\title{
Entrevista con Alan Baddeley
}

\author{
AGUSTIN ROMERO MEDINA \\ Universidad de Murcia \\ Area de Psicología Básica \\ Facultad de Filosofía, Psicología y CC. de la Educación
}

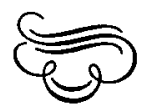

Uno de los investigadores actuales más importantes en psicología cognitiva es sin duda Alan Baddeley. Este profesor inglés dirige uno de los más prestigiosos centros de investigación en psicología, la Applied Psychology Unit (perteneciente al Medical Research Council) de Cambridge. Es un lugar donde más de 60 personas trabajan sobre más de 20 líneas de investigación con un denominador común: el interés en la investigación psicológica básica pero con repercusiones aplicadas.

Alan Baddeley es un prolífico investigador quien ha llevado a cabo multitud de trabajos, entre ellos, dos libros traducidos al castellano: Psicología de la memoria (1983) y Su memoria: Cómo conocerla y dominarla (1984), orientados fundamentalmente al estudio de los procesos de memoria. Sus investigaciones más destacadas condensan la versión más reciente de las propiedades activas o funcionales de la memoria a corto plazo (MCP) denominada «memoria opera-

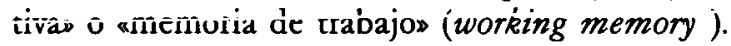

Esta breve entrevista, concedida durante una estancia del que escribe estas líneas en Cambridge (Mayo, 1987), se centra sobre este tema y alguna de sus implicaciones en temas específicos tratados por el entrevistado tales como la adquisición lectora y otros referentes al aprendizaje humano y cuestiones generales sobre la relación entre investigación psicológica básica y aplicada.

Pregunta: ¿La Applied Psychology Unit que usted dirige es sólo un centro de investigación?

Respuesta: Sí, tenemos estudiantes de investigación, estudiantes de doctorado y esta es la única labor de enseñanza que hacemos. Alguno de nosotros tienen que dar conferencias en universidades pero no trabajamos para enseñar sino para investigar.

P.: Sin embargo en España por lo general los profesores de universidad tenemos que investigar y enseñar..

R.: También es lo usual aquí, de modo que somos muy afortunados con tener este trabajo que nos permite dedicarnos a la investigación.

P.: ¿Cuáles son los temas o lineas de investigación en la Applied

Psychology Unit?

R.: Realmente son muy variados, desde la investigación en interacción hombre- 
ordenador, por ejemplo, al trabajo en psicología de la audición -diseño de todas las señales auditivas en la circulación- a problemas clínicos tales como la actividad cognitiva en la depresión.

P.: En España la psicología académica universitaria se ha distribuido en seis äreas de conocimiento. Por ejemplo, el área de Psicología Básica incluye temas de procesos cognitivos, psicología general, bistoria de la psicología, etc., pero actualmente tenemos un problema y es que la investigación en Psicología Básica es dificil de conectar con la sociedad, con las aplicaciones sociales. Usted investiga en un centro de psicología aplicada pero con un tipo de investigación básica. ¿Cómo lograr la comunicación entre dichos aspectos?

R.: Tenemos el mismo problema. Pienso que tenemos suerte con tener una tradición y un nombre como el de «Unidad de Psicología Aplicada» y la implicación es que debemos hacer cosas que se relacionen con el mundo, pero somos psicólogos fundamentalmente teóricos y así sentimos la necesidad de tender un puente sobre esta laguna y pienso que en psicología hoy esto no es difícil. Es posible hacer un trabajo teóricamente interesante y también útil. En otros temas puede ser difícil pero en psicología pienso que no es muy difícil. P.: En España bay también otras áreas de conocimiento en psicología - clinica, evolutiva, psicobiología, etc.-Pero la política universitaria en este ámbito ha sido la fragmentación.

R.: Sí, pienso que es una pena. Quizás sea importante construir puentes para que los individuos colaboren de forma que se evita esta fragmentación institucional.

P.: Un tema de interés para nosotros es el de la adquisición lectora, el aprendizaje de la lectura en niños normales. ¿Qué nos puede decir al respecto?

R.: Un colega y yo - Susan Gathercole- nos hemos dedicado al desarrollo de la lectura en niños normales a partir de algunos experimentos que hemos llevado a cabo en niños que fueron seleccionados por tener retraso en el lenguaje, según sus psicólogos educativos y se les enseñó separadamente. Cuando observamos a estos niños, la cuestión que nos interesaba era si no podíamos caracterizar dicho déficit como un déficit en la memoria operativa y en particular un déficit en algún componente del sistema del bucle articulatorio (articulatory loop). En general la respuesta fue que sí. En efecto, mostraban una amplitud de memoria muy alterada y, de hecho, su ejecución de memoria fue peor que la de los niños de igual edad lectora pero más jóvenes. El mejor discriminador entre dichos niños y los normales de igual inteligencia, fue la prueba en que se presentan al niño no-palabras que pueden variar en el número de sílabas y la tarea es simplemente volver a repetirlas. Lo que encontramos fue que estos niños estaban varios años por detrás de su edad cronológica en este test particular. Con esta prueba original hemos examinado a un gran número de niños -alrededor de 150 - que empezaron su escolaridad en el último mes de septiem. bre y también estamos estudiando su desarrollo del vocabulario debido a la posibilidad de que el sistema implicado en el aprendizaje de nuevo vocabulario tarda varios años en funcionar. Efectivamente, como resultado de nuestra investigación, hoy sabemos que nuestra tarea de repetición de no-palabras correlaciona altamente con vocabulario a los cuatro y a los cinco años de edad, predice la ejecución en vocabulario en esos niños al empezar su escolaridad y un año después, con lo cual podemos pensar que la memoria fonológica a corto plazo 
es importante para el aprendizaje del lenguaje a largo plazo. Quizás ayudará a identificar a niños que tienen semejante dificultad y nos dé así algunas pistas para ayudarles. Los datos preliminares también indican que quizás será un buen predictor de lectura.

P.: También estoy interesado en aprendizaje humano. La Psicología del Aprendizaje es un viejo tema muy tratado por el conductismo, y sin embargo pienso que el actual enfoque cognitivo no se interesa apenas por el aprendizaje, exceptuando quizás los desarrollos cognitivos del aprendizaje animal. En psicología cognitiva sé tratan temas de memoria, percepción, etc. pero, sobre todo, el aprendizaje humano apenas se considera como tal, ¿no lo ve usted asi?

R.: Pienso que esto está cambiando, parcialmente debido a los nuevos desarrolos de la Inteligencia Artificial. Hay un gran interés en las bases del aprendizaje según modelos de ordenador sobre el procesamiento en paralelo, los cuales a menudo usan términos conexionistas, los mismos de la vieja teoría del aprendizaje. Hay analogías entre estos modelos y algunos de los modelos de aprendizaje animal tales como el de Rescorla y Wagner.

Similarmente en un contexto aplicado, el aprendizaje por ejemplo en un contexto clínico o de reeducación de la lectura, a menudo se usan procedimientos tales como los desarrollados por los psicólogos operantes y a menudo usan diseños de caso único tales como los desarrollados por el condicionamiento operante. Así, me parece que la psicología cognitiva y la teoría del aprendizaje están llegando en conjunto a una gran flexibilidad y a la ampliación de la comprensión del aprendizaje más allá de escuelas particulares y con diferentes enfoques que ofrecer para diferentes cosas. De todos modos espero que en el futuro estas diferencias serán menores.

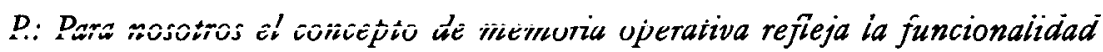
de la memoria a corto plazo (MCP). Según creo, la memoria operativa se ba investigado con una metodología de tareas dobles. ¿Piensa usted que es posible relacionar este enfoque con el de procesos automáticos $y$ controlados de Shiffrin y Schneider y con el de asignación de recursos de procesamiento de Navon y Gopher?

R.: Sí, pienso que, empezando por el enfoque de asignación de recursos de $\mathrm{Na}$ von y Gopher, se refiere a una metodología para dividir la capacidad. Implícitamente ellos asumen un solo canal. Pienso que el enfoque de la memoria operativa indica que eso es otra simplificación y probablemente ellos aceptarían también eso, por tanto yo vería la relación entre la memoria operativa y su interés en las técnicas para dividir la capacidad de procesamiento. Pienso que éstas son enteramente sensibles y apropiadas, pero su debilidad está en asumir sólo una capacidad y si uno tiene un límite de capacidad muy diferente de lo que quizás se necesita al emplear técnicas dentro de áreas particulares me parece que la memoria operativa ha sido un concepto más amplio pero quizás no tan preciso como para proyectar (mapping) la división de los recursos, por lo cual veo que son complementarios.

Respecto a Shiffrin y Schneider creo que ahora están interesados en un componente particular que sería parte del sistema de la memoria operativa y que comprenden su funcionamiento de la misma forma. De hecho, Schneider ha presentado muy recientemente un modelo de memoria operativa usando principios conexionistas que dice que no son incompatibles con otras formas de analizar los mismos problemas. Sostiene que la arquitectura del sistema de memo- 
ria operativa produce muchos de los fenómenos de la MCP. Tiene un trabajo sobre este tema en el volumen de 1987 de la serie de Bower sobre Advances in Psychology of Learning and Motivation.

P.: La pregunta anterior es debida a mi interés en la relación entre el. aprendizaje y los procesos automáticos y controlados. Precisamente usted señala en su reciente libro Working Memory que la memoria operativa es un proceso fundamental en el aprendizaje y la resolución de problemas. ¿Qué relación puede establecer entre el aprendizaje y la memoria operativa?

R.: Mi sospecha es que aprender unos items o material tiene que estar relacionado con un material que uno conoce previamente y para esto uno necesita atención o recursos con el fin de aprender. Es decir, el aprendizaje requiere atención y la atención confía en el ejecutivo central. Además, creo que la memoria operativa es un sistema «esclavo» que permite retener el material y manipularlo de forma que facilite el aprendizaje. Por tomar un ejemplo concreto, hemos llevado a cabo recientemente algunos experimentos con una paciente que tenía un gran déficit en MCP, un déficit en el almacenamiento fonológico. Una paciente italiana cuya capacidad para aprender a asociar el significado de palabras es del todo normal, pero si intentabas enseñarle una palabra no familiar no italiana, una palabra rusa, por ejemplo, su ejecución era desastrosamente mala, presumiblemente debido a que no puede retener una palabra rusa aislada debido a que su sistema de almacenamiento fonológico temporal no es adecuado. Si el material se presentaba visualmente entonces mostraba alguna evidencia de aprendizaje pero probablemente menor de lo normal.

\section{Referencias}

BADDEley, A. (1976). The psychology of memory. Nueva York: Harper \& Row. Trad. cast.: Psicología de la memoria. Madrid: Debate, 1983.

BADDELEY, A. (1982). Your memory: A user's quide. Londres: Sidgewich and Jackson, 1982. Trad. cast: Su memoria: Cómo conocerla y dominarla. Madrid: Debate, 1984.

Baddeley, A. (1986). Working memory. Oxford: Clarendon Press.

NAvON, D., y GOPHER, D. (1979). On the economy of the human-processing system. Psychological Review. 86, 214-255.

SHIFFRIN. R.M., y SCHNEIDER, W. (1977). Controlled and automatic human information processing. II. Perceptual learning, automatic attending, and a general theory. Psychological Review, 84. 127-190.

SCHNEIDER. W., y DETWEILER, M. (1987). A connectionist/control architecture for working memory. En G. H. Bower (Ed.): The psychology of leaming and motivation. Vol. 21. Nueva York: Academic Press. 